\title{
Basophils activated via TLR signaling may contribute to pathophysiology of type 1 autoimmune pancreatitis
}

\author{
Masato Yanagawa $^{1} \cdot$ Kazushige Uchida $^{1} \cdot$ Yugo Ando $^{1} \cdot$ Takashi Tomiyama $^{1}$. \\ Takashi Yamaguchi $^{1}$. Tsukasa Ikeura ${ }^{1}$. Toshiro Fukui ${ }^{1}$ Akiyoshi Nishio ${ }^{1}$. \\ Yoshiko Uemura $^{2} \cdot$ Takayuki Miyara $^{3}$ - Hiroyuki Okamoto ${ }^{4} \cdot$ Souhei Satoi $^{5}$. \\ Kazuichi Okazaki ${ }^{1}$
}

Received: 21 March 2017/Accepted: 5 September 2017/Published online: 18 September 2017

(c) The Author(s) 2017. This article is an open access publication

\begin{abstract}
Background Pathophysiology of type 1 autoimmune pancreatitis (AIP) is still unclear. We previously reported that M2 macrophages might play an important role in type 1 AIP. Recently, it has been reported that basophils regulate differentiation to M2 macrophages. In this study, we investigated basophils from the pancreatic tissue and peripheral blood of individuals with type 1 AIP.

Methods By using immunohistochemistry, we investigated basophils in pancreatic tissue from 13 patients with type 1 AIP and examined expression of toll-like receptors (TLRs) by these cells. Additionally, we obtained peripheral blood samples from 27 healthy subjects, 40 patients with type 1 AIP, 8 patients with alcoholic chronic pancreatitis, 10 patients with bronchial asthma, and 10 patients with atopic dermatitis, and analyzed activation of basophils by stimulating them with ligands of TLR1-9. We also compared TLR expression in basophils from the tissue and blood samples.
\end{abstract}

Kazuichi Okazaki

okazaki@hirakata.kmu.ac.jp

1 The Third Department of Internal Medicine, Division of Gastroenterology and Hepatology, Kansai Medical University, 2-5-1 Shinmachi, Hirakata, Osaka 573-1010, Japan

2 Department of Pathology, Kansai Medical University, Hirakata, Japan

3 The First Department of Internal Medicine, Division of Hematology, Respiratory Medicine and Rheumatology, Kansai Medical University, Hirakata, Japan

4 Department of Dermatology, Kansai Medical University, Hirakata, Japan

5 Department of Surgery, Kansai Medical University, Hirakata, Japan
Results Basophils were detected in pancreatic tissues from 10 of 13 patients with type 1 AIP. Flow cytometric analysis revealed that the ratios of basophils activated by TLR4 stimulation in type 1 AIP $(9.875 \pm 1.148 \%)$ and atopic dermatitis $(11.768 \pm 1.899 \%)$ were significantly higher than those in healthy subjects $(5.051 \pm 0.730 \%$; $P<0.05$ ). Levels of basophils activated by TLR2 stimulation were higher in seven type 1 AIP cases. Furthermore, stimulation of TLR2 and/or TLR4, which were expressed by basophils in pancreas, activated basophils in peripheral blood.

Conclusions Basophils activated via TLR signaling may play an important role in the pathophysiology of type 1 AIP.

Keywords Autoimmune pancreatitis - Basophil - TLR . M2 macrophage

$\begin{array}{ll}\text { Abbreviations } \\ \text { AIP } & \text { Autoimmune pancreatitis } \\ \text { LPSP } & \text { Lymphoplasmacytic sclerosing pancreatitis } \\ \text { IDCP } & \text { Idiopathic duct-centric pancreatitis } \\ \text { ICDC } & \text { International consensus diagnostic criteria } \\ \text { IgG4-RD } & \text { IgG4-related disease } \\ \text { ICOS } & \text { Inducible T cell co-stimulator } \\ \text { TGF- } \beta & \text { Transforming growth factor- } \beta \\ \text { TLR } & \text { Toll-like receptor } \\ \text { ACP } & \text { Alcoholic chronic pancreatitis } \\ \text { PAMPs } & \text { Pathogen-associated molecular patterns } \\ \text { DAMPs } & \text { Damage-associated molecular patterns } \\ \text { HMGB1 } & \text { High-mobility group box protein 1 }\end{array}$

Abbreviations

AIP Autoimmune pancreatitis

IDCP Idiopathic duct-centric pancreatitis

International consensus diagnostic criteria

IgG4-RD IgG4-related disease

Inducible $T$ cell co-stimulator

Transforming growth factor- $\beta$

Toll-like receptor

Alcoholic chronic pancreatitis

PAMPs Pathogen-associated molecular pattern

HMGB1 High-mobility group box protein 1 


\section{Introduction}

Autoimmune pancreatitis (AIP) is characterized by chronic pancreatic inflammation caused by immune mechanisms $[1,2]$. Steroid treatment is remarkably effective for AIP, which may be sometimes difficult to be differentiated from pancreatic carcinoma [3]. The first report of AIP was a case of chronic pancreatitis with hypergammaglobulinemia that was published in 1961 [4]. Thirty years later, this pathological syndrome was defined as lymphoplasmacytic sclerosing pancreatitis (LPSP) [5], and proposed to be referred to as "autoimmune pancreatitis" by Yoshida et al. in 1995 [6]. Hamano et al. revealed that serum IgG4 levels were elevated in patients with AIP [7], and this condition is currently classified as type 1 AIP. On the other hand, Notohara et al. reported a different pathological condition, which was defined as idiopathic duct-centric pancreatitis (IDCP), which is classified as type 2 AIP [8]. In 2011, the international consensus diagnostic criteria (ICDC) for AIP classified two subtypes of AIP, type 1 and type 2 [9]. Type 1 AIP is recognized as the pancreatic manifestation of IgG4-related disease (IgG4-RD), which involves various organs, including the pancreas, salivary glands, thyroid glands, lungs, bile ducts, kidneys, retroperitoneum, lymph nodes, and others [10-13]. In serology, most patients with type 1 AIP exhibit elevated serum IgG4 levels [7], and some of them show elevated serum $\operatorname{IgE}[14,15]$. Histological features of type 1 AIP include diffuse lymphoplasmacytic and eosinophilic infiltrates, storiform fibrosis, obliterative phlebitis, and the infiltration of a large number of IgG4-positive plasma cells [9]. Regulatory $\mathrm{T}$ cells and $\mathrm{T}$ helper 2 (Th2) cytokines, such as IL-4, IL-5, and IL-13, are considered to contribute to pathophysiology of type 1 AIP $[16,17]$. We have previously reported that the number of regulatory $\mathrm{T}$ cells is increased in peripheral blood and infiltrated target organs of type 1 AIP [18]. This particularly concerns inducible $\mathrm{T}$ cell co-stimulator (ICOS)-positive regulatory $\mathrm{T}$ cells, which promote $\mathrm{IgG} 4$ production via IL-10, and ICOS-negative regulatory T cells, which induce fibrosis via transforming growth factor (TGF)- $\beta$ [18]. Furthermore, the pathophysiology of type 1 AIP is characterized by Th2-dominant reaction, but its mechanism has not yet been elucidated [19]. Our latest report suggested that M2 macrophages play an important role in the development of type 1 AIP [20]. IL-33 produced by M2 macrophages also promotes $\mathrm{Th} 2$ cytokine production conferring Th2-dominant pathophysiology in IgG4-RD [21]. Additionally, numerous macrophages were identified in AIP-affected tissue [22]. On the other hand, basophils were found to be required for the transformation of inflammatory monocytes into M2 macrophages in allergic disease [23]. However, the role of basophils in type 1 AIP is not clear.

In this study, to clarify the involvement of basophils activated via the toll-like receptor (TLR) signaling pathway in type 1 AIP, we investigated infiltration of TLR-expressing basophils in pancreatic tissue and their reactivity upon TLR stimulation in patients with type 1 AIP.

\section{Methods}

In our experiments, we initially sought to confirm the presence of basophils in pancreatic tissue and then investigated whether they expressed TLRs. Furthermore, we examined the responses of basophils to TLR stimulation using peripheral blood mononuclear cells from patients with type 1 AIP.

\section{Subjects}

For immunohistochemical analysis, we examined pancreatic tissue samples from 13 patients with type 1 AIP ( 6 men and 7 women; mean age 66 years; range 56-76 years).

We also conducted immunofluorescence analysis in 10 of 13 cases. All cases were surgically treated at the Kansai Medical University Hospital between 2001 and 2013, and in all cases, pancreatic ductal adenocarcinoma was suspected before the operation. The patients were histopathologically diagnosed with type 1 AIP. For comparison, we also examined pancreatic tissue samples from 10 patients with alcoholic chronic pancreatitis (ACP). All the $10 \mathrm{ACP}$ patients underwent surgical operation.

For flow cytometry analysis, we recruited 27 healthy subjects (17 men and 10 women; mean age 66 years; range $42-83$ years), 40 patients with type 1 AIP, who had not received corticosteroid treatment (29 men and 11 women; mean age 65 years; range $44-83$ years), 8 patients with ACP (6 men and 2 women; mean age 57 years; range 39-69 years), 10 patients with bronchial asthma, who did not receive oral medication (6 men and 4 women; mean age 52 years; range 39-74 years), and 10 patients with atopic dermatitis, who did not receive oral medication $(8$ men and 2 women; mean age 49 years; range $27-77$ years). Patient profiles are listed in Table 1. There was no difference in gender distribution between type 1 AIP and healthy subjects. The mean ages of bronchial asthma patients and atopic dermatitis patients were lower than those of patients with type 1 AIP and healthy subjects $(P<0.05)$. However, ages of patients with type 1 AIP, ACP, and healthy subjects were not significantly different. The mean values of serum IgG4 levels in type 1 AIP patients were significantly higher 
Table 1 Demographics and baseline characteristics of the patients in the present study

\begin{tabular}{|c|c|c|c|c|c|}
\hline Patients characteristic & $\begin{array}{l}\text { Healthy subject } \\
(n=27)\end{array}$ & $\begin{array}{l}\text { Type } 1 \text { AIP } \\
(n=40)\end{array}$ & $\begin{array}{l}\text { Alcoholic chronic } \\
\text { pancreatitis }(n=8)\end{array}$ & $\begin{array}{l}\text { Bronchial asthma } \\
(n=10)\end{array}$ & $\begin{array}{l}\text { Atopic dermatitis } \\
(n=10)\end{array}$ \\
\hline Gender (male/female) & $16 / 11$ & $29 / 11$ & $6 / 2$ & $6 / 4$ & $8 / 2$ \\
\hline Age (years, mean $\pm \mathrm{SE}$ ) & $65.63 \pm 10.43$ & $65.03 \pm 10.70$ & $57.00 \pm 3.82$ & $51.70 \pm 13.38^{*}$ & $49.00 \pm 17.31^{*}$ \\
\hline $\begin{array}{l}\text { Serum IgG4 levels }(\mathrm{mg} / \mathrm{dL} \text {, } \\
\quad \text { mean } \pm \mathrm{SE})\end{array}$ & $\begin{array}{l}32.90 \pm 4.15 \\
\quad(n=13)\end{array}$ & $\begin{array}{l}465.85 \pm 67.08 \\
\quad(n=40)^{*}\end{array}$ & $48.30 \pm 12.36(n=8)$ & $\begin{array}{l}37.43 \pm 6.75 \\
\quad(n=8)\end{array}$ & $\begin{array}{l}124.00 \pm 33.03 \\
(n=8)\end{array}$ \\
\hline
\end{tabular}

There was no difference in gender distribution between type 1 AIP and healthy subjects. The mean ages of bronchial asthma patients and atopic dermatitis patients were lower than those of patients with type 1 AIP and healthy subjects $(P<0.05)$. However, ages of patients with type 1 AIP, ACP, and healthy subjects were not significantly different. The mean values of serum IgG4 levels in type 1 AIP patients were significantly higher than in other groups $(P<0.05)$

$N S$ no significant differences compared with healthy or other disease controls

$* P<0.05$ significant differences compared with healthy controls

than in other groups $(P<0.05$; Table 1$)$. The numbers of basophils in the peripheral blood were not different in patients with type 1 AIP, ACP, and healthy subjects. All patients with type 1 AIP were diagnosed with AIP by the ICDC [9].

This study was approved by the ethics committee of the Kansai Medical University.

\section{Immunohistochemistry and antibodies}

We used mouse antibodies specific to human basophils (2D7; Abcam, Cambridge, UK) in order to identify basophils by immunohistochemical staining. The fields of view with the highest density of basophils were evaluated, and the numbers of basophils in three different fields of $1 \mathrm{~mm}^{2}$ each were summed. Next, we examined the expression of TLRs on infiltrated basophils using double-immunofluorescence staining of pancreatic tissue from patients with type 1 AIP with mouse antibodies specific to human basophils and rabbit antibodies specific to human TLR1-9.

For immunohistochemical staining, 4- $\mu \mathrm{m}$-thick formalin-fixed and paraffin-embedded sections were prepared, deparaffinized, and rehydrated using xylene and solutions with gradually decreasing concentrations of alcohol. Endogenous peroxidase activity was blocked for immunohistochemical sections using a treatment with $3 \%$ $\mathrm{H}_{2} \mathrm{O}_{2}$ in methanol for $10 \mathrm{~min}$ at room temperature. After washing in distilled water, the slides were treated with $0.05 \%$ trypsin (Abcam) in PBS ( $\mathrm{pH} \mathrm{7.3)} \mathrm{for} 1 \mathrm{~h}$ at room temperature to enhance antigenicity. All slides were incubated for $10 \mathrm{~min}$ in Protein Block Serum-Free (Dako, Kyoto, Japan). After protein blocking, the slides were incubated overnight at $4{ }^{\circ} \mathrm{C}$ with mouse primary antibodies specific to human basophils (2D7). The primary antibodies were dissolved in Signal Enhancer HIKARI (Nacalai Tesque, Kyoto, Japan). The slides were then incubated with secondary antibodies using a Chem Envision kit/HRP
(Dako) following the manufacturer's instructions. Finally, antibody binding was detected using 3,3'-diaminobenzidine (Dojindo, Kumamoto, Japan). Sections were counterstained with hematoxylin. Negative controls were evaluated by replacing the primary antibodies with similarly diluted non-immunized serum. Images were obtained with a light microscope (DP73; Olympus, Tokyo, Japan).

In the double-immunofluorescence method, the slides were incubated with mouse antibodies specific to human basophils (2D7) and rabbit antibodies specific to human TLR1-9 (TLR1: AbFrontier, Seoul, Korea; TLR2: Novus, Littleton, CO, USA; TLR3: Novus; TLR4: StressMarq Biosciences, Victoria, Canada; TLR5: AbFrontier; TLR6: AbFrontier; TLR7: AbFrontier; TLR8: AbFrontier; TLR9: GeneTex, Irvine, CA, USA) as the primary antibodies overnight at $4{ }^{\circ} \mathrm{C}$. The slides were then incubated with Alexa Fluor 546 anti-mouse immunoglobulins and Alexa Fluor 488 anti-rabbit immunoglobulins (Molecular Probes, Eugene, OR, USA) as secondary antibodies for $1 \mathrm{~h}$ at room temperature, protected from light. After incubation with the primary and secondary antibodies, slides were mounted with VECTASHIELD Mounting Medium and 4',6-diamidino-2-phenylindole (DAPI; Vector Laboratories, Burlingame, CA, USA) to counterstain nuclei and preserve fluorescence. Negative controls were evaluated by replacing the primary antibodies with similarly diluted non-immunized serum. Images were obtained with an immunofluorescence microscope (DP73; Olympus). Additionally, we were able to obtain both the pancreatic tissues and blood from 5 out of the 10 patients, whose samples were used for immunofluorescence analysis, so we examined the relationship of each TLR.

\section{Flow cytometry and antibodies}

We analyzed the activation of basophils using Allergenicity Kits (Beckman Coulter Company, Brea, CA, USA). The 
analysis of whole-blood specimens collected with EDTA as an anticoagulant was performed according to the manufacturer's instructions. Whole-blood specimens were stimulated with TLR1-9 ligands Pam ${ }_{3}$ CSK4 (400 ng/mL, InvivoGen, San Diego, CA, USA), HKLM $\left(10^{8}\right.$ cells $/ \mathrm{mL}$, InvivoGen), poly:IC (50 $\mu \mathrm{g} / \mathrm{mL}$, Sigma, St. Louis, MO, USA), LPS ( $1 \mu \mathrm{g} / \mathrm{mL}$, Sigma), FLA-ST $(10 \mu \mathrm{g} / \mathrm{mL}$, InvivoGen), FSL-1 (5 $\mathrm{g} / \mathrm{mL}$, InvivoGen), Imiquimod (5 $\mu \mathrm{g} /$ $\mathrm{mL}$, InvivoGen), ssRNA40/LyoVec (5 $\mathrm{g} / \mathrm{mL}$, InvivoGen) or ODN2006 $(10 \mu \mathrm{g} / \mathrm{mL}$, AdipoGen, San Diego, CA, USA), respectively. First, we mixed $100 \mu \mathrm{L}$ of wholeblood specimens, activation solution, and three color reagents (CRTH2-FITC: BM16, CD203c-PE: 97A6, and CD3-PC7: UCHT1) with $10 \mu \mathrm{L}$ of a TLR ligand or PBS in each tube. Activation solution is an optimized calciumenriched buffer to activate basophils in vitro. Then, we incubated each tube for $15 \mathrm{~min}$ at $37{ }^{\circ} \mathrm{C}$ in the atmosphere of $95 \%$ air $/ 5 \%$ carbon dioxide, protected from light. After staining, we added $2 \mathrm{~mL}$ of Fix-and-Lyse solution, which included the Allergenicity Kit, to remove erythrocytes interference. We incubated the tubes for another $10 \mathrm{~min}$ at room temperature, protected from light. We then centrifuged the tubes for $5 \mathrm{~min}$ at $200 \mathrm{~g}$ and aspirated the supernatant twice. Finally, we re-suspended the cells in $0.3 \mathrm{~mL}$ of PBS with $0.1 \%$ formaldehyde and analyzed them using flow cytometry. Flow cytometric analysis was performed by FACS Calibur II (BD Biosciences, Franklin Lakes, NJ, USA).

We counted the ratio of activated basophils in accordance with the manufacturer's instructions for the Allergenicity Kit (Fig. 1).

First, lymphocytes and monocytes were isolated, whereas CD3-positive events were excluded. Next, basophils were isolated as CD294 (CRTH2)-positive events from lymphocytes and monocytes. Finally, we drew line A so that $2-5 \%$ of basophils were included above it, and defined them as CD203c-positive basophils, because 2-5\% of basophils are activated basophils in normal conditions. CD203c has been demonstrated to be a specific activation marker of basophils. Additionally, we compared the ratio of CD203c-positive basophils before and after TLR stimulation in each group.

\section{Statistical analysis}

In immunohistochemistry and flow cytometry experiments, quantitative data are presented as the mean \pm standard error of the mean (SE). The Tukey-Kramer test was used to compare quantitative values in flow cytometry experiments. Differences were considered to be statistically significant when the value of $P$ was less than 0.05 $(* P<0.05)$.

\section{Results} Identification of basophils in pancreatic tissue
from patients with type 1 AIP

Basophils (2D7-positive cells) were detected in the pancreatic tissue samples from 10 of 13 patients with type 1 AIP cases by immunohistochemistry (Fig. 2). In contrast, 2D7-positive cells were not detected in the pancreatic tissue from patients with ACP cases (Fig. 3). The average number of 2D7-positive cells was $8.615 \pm 2.528$, measured in three different $1-\mathrm{mm}^{2}$ fields of view in samples from each of the 13 cases (Fig. 4).

\section{TLR expression by basophils from pancreatic tissue of patients with type 1 AIP}

We identified 2D7-positive cells (red; Fig. 5a, e), TLR2positive cells (green; Fig. 5b), and TLR4-positive cells (green; Fig. 5f), and examined them in the merged view (Fig. 5d, h), overlaid with DAPI nuclear staining (Fig. 5c, g). 2D7-positive cells expressed TLR2 in 2 of 10 cases, TLR 4 in 6 of 10 cases, and both TLR 2 and TLR 4 in 2 of 10 cases. 2D7-positive cells were not detected among other TLR-positive cells (Table 2).

\section{Relationship between TLR expression by basophils from pancreatic tissue and activation of peripheral blood basophils upon stimulation of TLR1-9}

We examined the relationship between TLR expression by basophils from pancreatic tissue and activation of basophils in the peripheral blood by stimulating them with TLR1-9 ligands. We found that the levels of activated basophils in the peripheral blood were elevated by TLR2 stimulation in the two patients (case 1 and 2) that exhibited TLR2-positive basophils in the pancreatic tissue. In one case (case 3), which exhibited both TLR2- and TLR4-positive basophils in the pancreatic tissue, levels of activated basophils in the peripheral blood of this patient were elevated by only both TLR2 and TLR4 stimulation. In the two cases (case 5 and 6) that exhibited TLR4-positive basophils in the pancreatic tissue, levels of activated basophils in peripheral blood were elevated by TLR4 stimulation, but not other types of TLR (Table 3). Thus, in patients with type 1 AIP, stimulation of the TLR that is expressed on the basophils in the pancreatic tissue seems to activate circulating basophils.

\section{Ratios of basophils activated by TLR1-9 stimulation}

In the absence of TLR stimulation, CD203c expression levels in healthy subjects $(265.897 \pm 34.449)$, patients 
Fig. 1 Identification of resting and activated basophils from peripheral blood by flow cytometry. All leukocytes were stained with CD3, CD294, and CD203c. All leukocytes formed discrete FSC/SSC populations after fixation and lysis (a). CD3positive events (R1) were removed due to the isolation of lymphocytes and monocytes (b). Basophils were defined as CD294 (CRTH2)-positive events (R2) (c). CD203c is a specific marker of activated basophils. Line A was drawn so that $2-5 \%$ of basophils were included above it, and we defined them as activated basophils. We compared the percentage of basophils above line A before and after the stimulation (d). These experiments were performed using the Allergenicity Kit (Beckman Coulter Company, Brea, CA, USA)
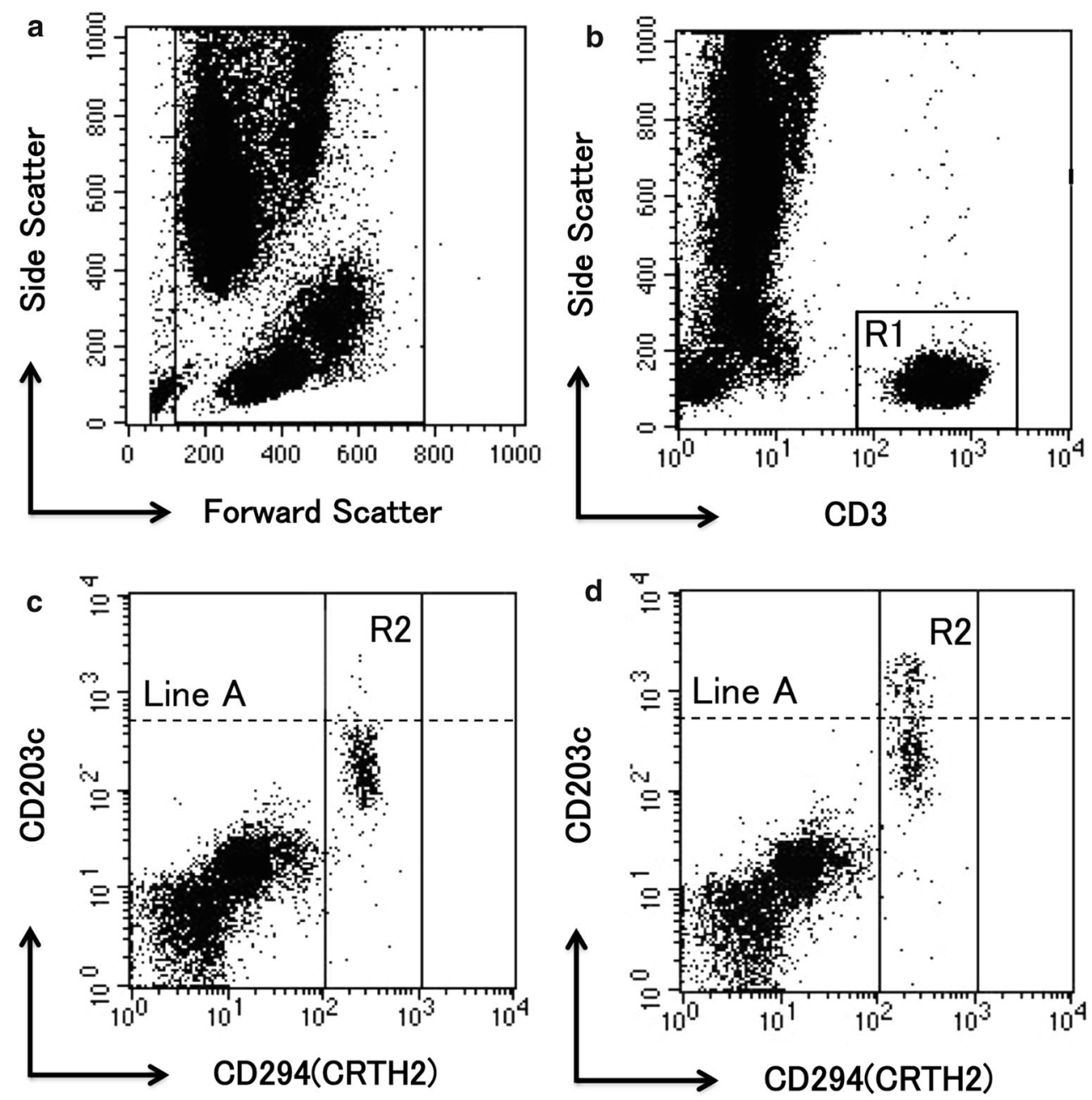

with type 1 AIP $(238.364 \pm 17.130), \quad$ ACP (235.84 \pm 44.43$)$, bronchial asthma (251.546 \pm 50.610$)$, and atopic dermatitis $(194.674 \pm 49.491)$ in mean fluorescence intensity (MFI) were not significantly different, and the ratio of activated basophils in healthy subjects $(2.758 \pm 0.144 \%)$, patients with type 1 AIP $(3.167 \pm 0.156 \%)$, patients with ACP $(2.256 \pm 0.133 \%)$, bronchial asthma $(3.489 \pm 0.282 \%)$, and atopic dermatitis $(2.946 \pm 0.118 \%)$ were also not significantly different (data not shown). However, the ratios of basophils activated by TLR4 stimulation in the blood of patients with type 1 AIP $(9.875 \pm 1.148 \%)$ and atopic dermatitis $(11.768 \pm 1.899 \%)$ were significantly higher than that in healthy subjects $(5.051 \pm 0.730 \% ; P<0.05)$. Furthermore, the ratio of TLR4-activated basophils in patients with type 1 AIP were not significantly elevated compared to ACP, but tended to be elevated $(P=0.08)$. In 7 of the 40 patients with type 1 AIP, CD203c expression levels were elevated by TLR2 stimulation, although the effect did not reach statistical significance. In addition, CD203c expression levels following stimulation of other TLRs were not significantly different between the groups (Fig. 6).

\section{Discussion}

In this study, we identified the presence of basophils in pancreatic tissue of patients with type 1 AIP. We also found that the ratio of basophils activated by TLR4 signaling in the peripheral blood of patients with type 1 AIP was significantly higher than that in healthy subjects. In addition, the ratio of basophils activated by TLR 2 signaling was elevated in 7 of 40 cases of type 1 AIP (Fig. 6). Remarkably, we revealed that stimulation of the type of TLR that was expressed on infiltrated basophils in type 1 AIP-affected pancreatic tissue also activated circulating basophils (Table 3). On the other hands, basophils were not detected in the pancreatic tissue with ACP, and the ratio of basophils activated by TLR signaling in the peripheral blood of patients with ACP were not significantly different as compared with healthy subjects. Furthermore, the ratio 

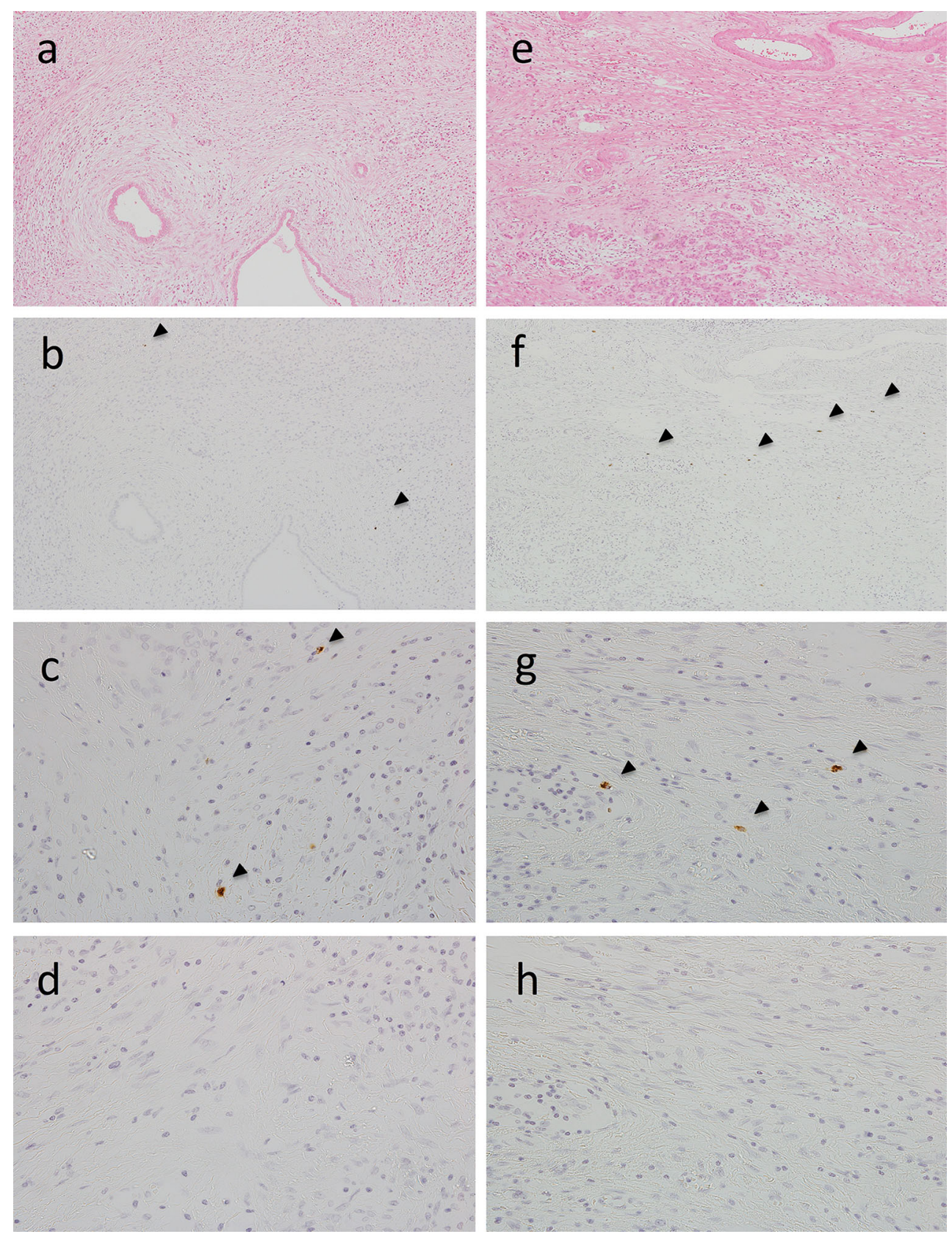

Fig. 2 H\&E staining (a, e), immunohistochemical findings for basophils [low magnification view, $\times 100](\mathbf{b}, \mathbf{f})$, [high magnification view, $\times 400$ ] $(\mathbf{c}, \mathbf{g})$, and negative controls [high magnification view,

$\times 400](\mathbf{d}, \mathbf{h})$ in pancreatic tissue samples of two cases with type 1 autoimmune pancreatitis (AIP). Infiltration of basophils was confirmed by using mouse anti-basophil antibodies (2D7)

of TLR4-activated basophils in patients with type 1 AIP tended to be elevated compared to ACP. Therefore, we speculated that basophils activated by TLR signaling were important in the development of type 1 AIP.

Basophils comprise less than $1 \%$ of human peripheral blood leukocytes and can live only for a few days in the non-activated condition [24]. However, it has been shown that 10-times higher levels of Th2 cytokines such as IL4 and IL13 are immediately produced compared with those in lymphocytes, even when a small percentage of basophils

are activated $[25,26]$. They are generally not present in normal tissue and become recruited to affected tissue sites only under certain conditions, for example, during allergic reactions [27]. It has been shown that basophils act as initiators of inflammatory cell recruitment during the progression of IgE-mediated chronic allergic inflammation [28]. It has also been reported that basophils in skin lesions from atopic dermatitis were detected in approximately $60 \%$ of patients; however, the cell density was low as compared with patch-tested lesions [29]. Patients with type 1 AIP 

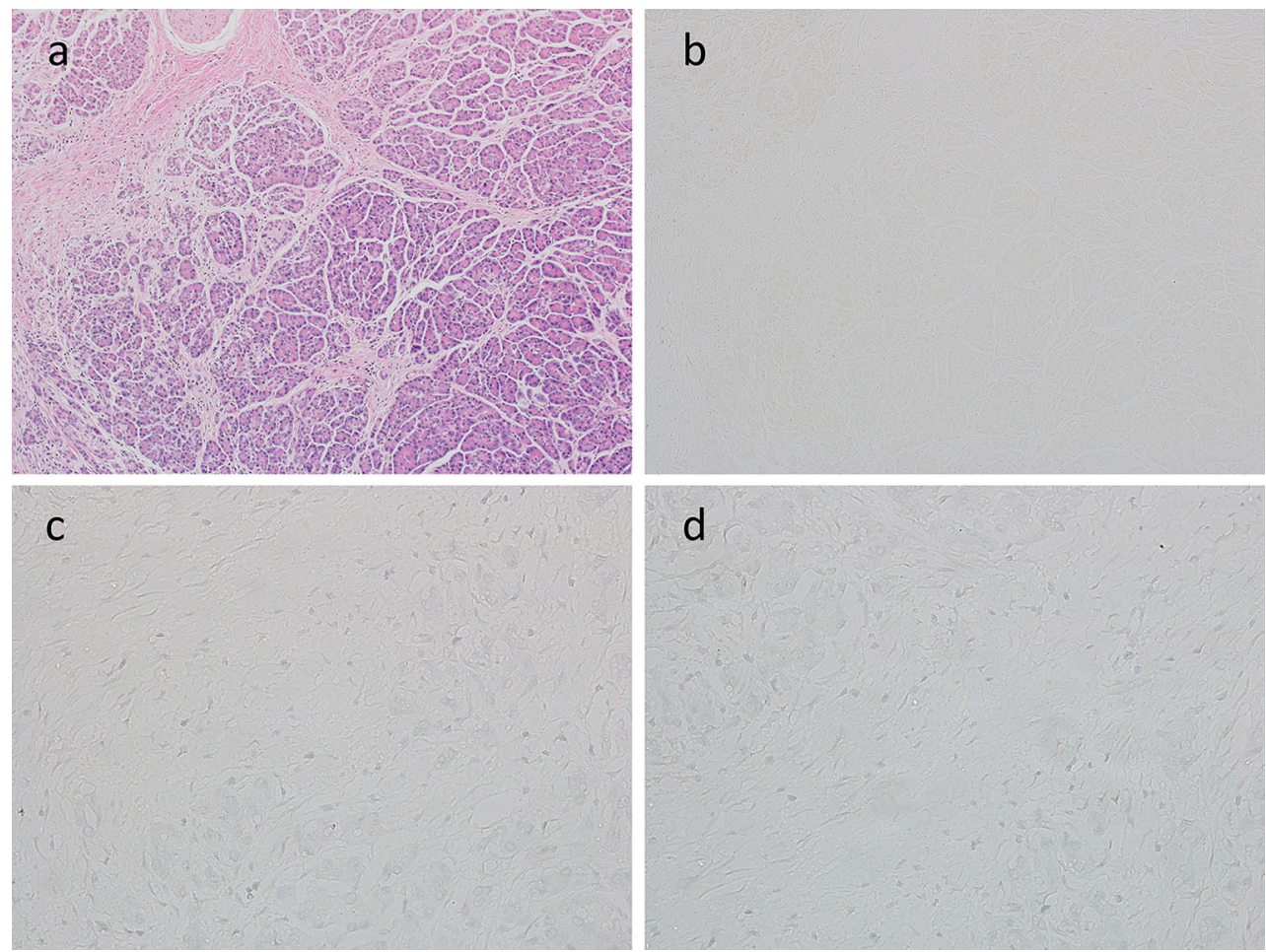

Fig. 3 H\&E staining (a), immunohistochemical findings for basophils [low magnification view, $\times 100$ ] (b), [high magnification view, $\times 400$ ] (c), and negative controls [high magnification view, $\times 400$ ] (d) in a pancreatic tissue sample with alcoholic chronic pancreatitis $(\mathrm{ACP})$

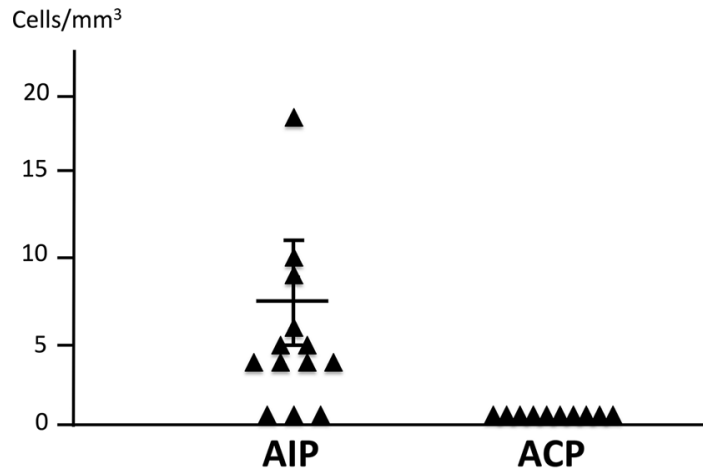

Fig. 4 Comparison of the total number of basophils in three different $1-\mathrm{mm}^{2}$ fields of view in pancreatic tissue samples from patients with type 1 AIP or alcoholic chronic pancreatitis (ACP). The average number of basophils in samples from patients with type 1 AIP was $8.615 \pm 2.528(n=13)$. However, basophils were not detected in the pancreatic tissue of patients with ACP $(n=10)$

have frequent complications of allergic conditions, such as bronchial asthma or allergic dermatitis, characterized by elevated serum IgE and eosinophilia [21, 30]. In this study, we identified basophils in pancreatic tissue samples from 10 of 13 patients with type 1 AIP (Fig. 4), but the cell density was low. This finding indicates that pathophysiology of type 1 AIP may be similar to that of an allergic disease, such as atopic dermatitis. We, therefore, speculate that basophils infiltrating in the tissues might play an important role in the Th2-dominant condition of type 1 AIP.

Basophil-derived IL-4 is considered to be involved in the recruitment of monocytes and their differentiation to M2 macrophages in allergic skin [23]. Macrophages are composed of at least two distinct groups of M1 and M2 phenotypes. M1 macrophages elicit an inflammatory responses and play a central role in host defense against bacterial and viral infections, whereas M2 macrophages play roles in anti-inflammatory responses, reactions to parasitic infections, as well as in tissue repair and remodeling [31]. M2 macrophages induced by IL-4 are involved in fibrosis as they produce IL-10 and CCL18 [32]. Notohara et al. reported that CD163-positive spindle-shaped macrophages contribute to LPSP manifestations, such as storiform fibrosis in type 1 AIP [33]. We previously reported that macrophages (especially CD163-positive cells), stimulated via TLR signaling pathways, might play an important role in pancreatic tissue from patients with type 1 AIP [20]. Furthermore, the number of M2 macrophages in salivary glands of individuals with IgG4-RD was significantly higher than that in healthy subjects, and the distribution of IL-10 and CCL18 closely paralleled that of M2 macrophages [34]. These findings suggest that basophils induce M2 macrophages, which, in turn, may contribute to fibrosis in the pancreatic tissue affected by type 1 AIP. 

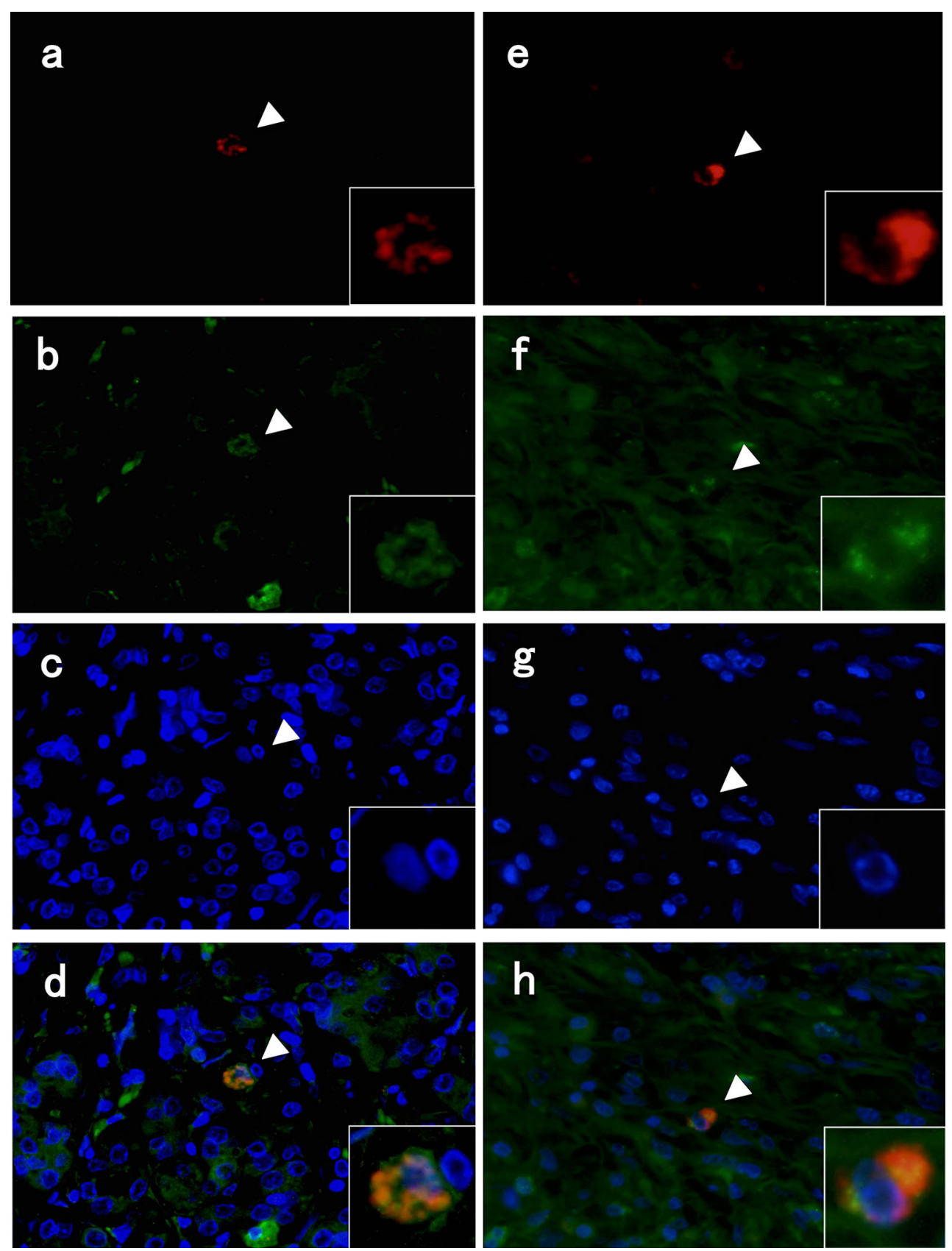

Fig. 5 Double-immunofluorescence staining for basophils, TLR2, and TLR4 in pancreatic tissue of patients with type 1 autoimmune pancreatitis. Images show staining for basophils (2D7; red, a, e),

TLR2 (green, b), TLR4 (green, f), and 4',6-diamidino-2-phenylindole (DAPI, blue, c, g). Merged image of basophils and TLR2 (d)/TLR4 (h)

It is known that Th2 cytokine production is elevated in pancreatic tissue of type 1 AIP patients, but the mechanism of this phenomenon has not yet been clarified [35]. It was recently demonstrated that endogenous IL-33 promoted Th2 cytokine production and induced allergic inflammation during allergic airway inflammation [36]. IL-33 produced by $\mathrm{M} 2$ macrophages promoted Th2 cytokine production via IL-33 receptor activation and contributed to Th2-dominant pathophysiology in IgG4-RD [37]. Another study revealed

that basophils rapidly produced large amounts of IL-4 in response to various stimuli, including activation by ligands of TLRs, in innate-type allergy, and could induce differentiation of Th2 cells without cross-linkage of FceRI [38]. On the basis of these reports and our present data, we speculate that basophils are important cells in Th2-dominant pathophysiology of type 1 AIP.

Additionally, we identified the presence of TLR2- and/ or TLR4-positive basophils in pancreatic tissue from 
Table 2 TLR expression on basophils infiltrated into the pancreatic tissue with type 1 AIP

\begin{tabular}{|c|c|c|c|c|c|c|c|c|c|c|c|}
\hline Case no. & Age & Gender & TLR1 & TLR2 & TLR3 & TLR4 & TLR5 & TLR6 & TLR7 & TLR8 & TLR9 \\
\hline 1 & 63 & M & - & + & - & - & - & - & - & - & - \\
\hline 2 & 71 & $\mathrm{~F}$ & - & + & - & - & - & - & - & - & - \\
\hline 3 & 78 & $\mathrm{~F}$ & - & + & - & + & - & - & - & - & - \\
\hline 4 & 67 & M & - & + & - & + & - & - & - & - & - \\
\hline 5 & 67 & M & - & - & - & + & - & - & - & - & - \\
\hline 6 & 56 & M & - & - & - & + & - & - & - & - & - \\
\hline 7 & 57 & $\mathrm{~F}$ & - & - & - & + & - & - & - & - & - \\
\hline 8 & 60 & $\mathrm{~F}$ & - & - & - & + & - & - & - & - & - \\
\hline 9 & 60 & $\mathrm{~F}$ & - & - & - & + & - & - & - & - & - \\
\hline 10 & 71 & M & - & - & - & - & - & - & - & - & - \\
\hline
\end{tabular}

Table 3 Relationship of the TLR expression on basophils in the peripheral blood and the pancreas from type 1 AIP

\begin{tabular}{lllllrl}
\hline Case no. & Age & Gender & Serum IgG4 (mg/dL) & $\begin{array}{l}\text { TLR expression on } \\
\text { basophils in the pancreas }\end{array}$ & Activated ratio of basophils in the peripheral blood (\%) \\
\cline { 6 - 7 } & & & & TLR2 & TLR4 \\
\hline 1 & 63 & M & 153 & TLR2 & 60.1 & 3.9 \\
3 & 71 & F & 119 & TLR2 & 6.0 & 4.8 \\
5 & 78 & F & 65.9 & TLR2, & 5.4 & 9.4 \\
6 & 67 & M & 20 & TLR4 & 3.0 & 10.2 \\
\hline
\end{tabular}

Case no. is the same as those in Table 2. No. 4, 7-10 have not been tested by flow cytometry

In case 1 and case 2, which exhibited TLR2-positive basophils in the pancreatic tissue, levels of activated basophils in the peripheral blood of these patients were elevated by TLR2 stimulation. In case 3, which exhibited both TLR2- and TLR4-positive basophils in the pancreatic tissue, levels of activated basophils in peripheral blood were elevated by only both TLR2 and TLR4 stimulation. In case 5 and case 6 , that exhibited TLR4-positive basophils in the pancreatic tissue, levels of activated basophils in peripheral blood were elevated by TLR4 stimulation, but not other types of TLR

patients with type 1 AIP (Table 2). We could confirm that basophils express TLR2 or TLR4 in this study, but some researchers reported that antigen presenting cells such as dendritic cells, macrophages, B cells, and $\mathrm{T}$ cells express TLR2 or TLR4 [39-41]. The presence of basophils expressing different TLRs (TLR2 and/or TLR4) in the pancreatic tissue suggests that pathophysiology of type 1 AIP may be caused by a heterogeneous inflammatory condition.

There was no difference of the clinical data (blood test results, location of pancreatic swelling, or extent of involvement of other organs) between the TLR2-positive group and TLR4-positive group in AIP patients. There was also no correlation between the ratio of basophils activated by TLR signaling and serum IgG4 and IgE levels (data not shown). However, Watanabe et al. reported that basophils activated via TLR2 or TLR4 signaling enhanced the production of $\mathrm{IgG} 4$ through a BAFF-mediated signaling pathway in IgG4-RD [42]. On the other hand, there was no correlation between serum IgG4 levels and the number of IgG4-positive cells in AIP patients (data not shown). Paik et al. also reported that there was no correlation between serum and tissue IgG4 levels [43]. Basophils that infiltrated into pancreatic tissue of patients with type 1 AIP may also contribute to IgG4 production.

TLRs are pattern recognition receptors that recognize pathogen-associated molecular patterns (PAMPs) or damage-associated molecular patterns (DAMPs). PAMPs are associated with microbial pathogens, whereas DAMPs are linked to cell components, which are released during cell damage or death, to induce the innate immune response [43]. It is well known that PAMPs derived from abundant gut bacteria are transferred to the serum and bone marrow, where they promote systemic innate immunity [44]. It is also possible that DAMPs activate basophils in pancreatic tissue from patients with type 1 AIP. DAMPs of TLR2 and TLR4 include biglycan, high-mobility group box protein 1 (HMGB1), heat shock protein 70, fibronectin, and others [45]. They have been shown to play roles in the induction of experimental pancreatitis [46]. HMGB1, released by necrotic acinar cells, induces tissue injury and inflammation via TLR4 activation $[47,48]$. As a result of acinar cell necrosis, HMGB1 may activate basophils via TLR4 in pancreatic tissue of type 1 AIP patients. HMGB1 and selfnucleic acid complexes are also involved in the development of chronic inflammatory disease [49, 50]. Thus, 


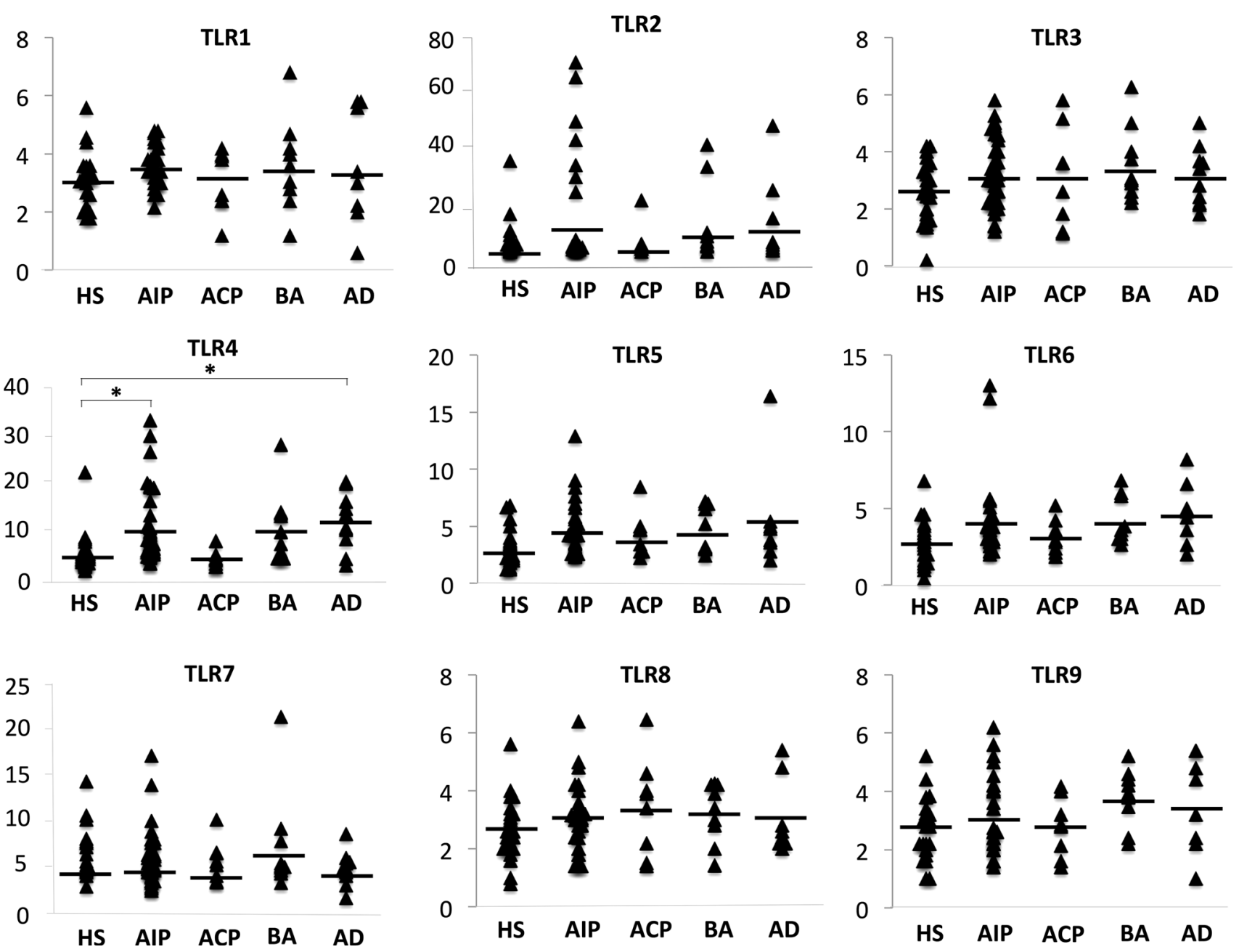

Fig. 6 The ratio of basophils activated by the stimulation of TLR1TLR9. We analyzed peripheral basophils in healthy subjects $(n=27)$, patients with type 1 autoimmune pancreatitis (AIP, $n=40$ ), patients with alcoholic chronic pancreatitis (ACP, $n=8$ ), patients with bronchial asthma $(n=10)$, and patients with atopic dermatitis $(n=10)$. Basophils were stimulated with the TLR1-9 ligands, Pam ${ }_{3}$ CSK4 (400 ng/mL, InvivoGen, San Diego, CA, USA), HKLM $\left(10^{8}\right.$ cells $/ \mathrm{mL}$, InvivoGen), poly:IC $(50 \mu \mathrm{g} / \mathrm{mL}$, Sigma, St.

Louis, MO, USA), LPS ( $1 \mu \mathrm{g} / \mathrm{mL}$, Sigma), FLA-ST $(10 \mu \mathrm{g} / \mathrm{mL}$, InvivoGen), FSL-1 (5 $\mu \mathrm{g} / \mathrm{mL}$, InvivoGen $)$, imiquimod $(5 \mu \mathrm{g} / \mathrm{mL}$, InvivoGen), ssRNA40/LyoVec (5 $\mu \mathrm{g} / \mathrm{mL}$, InvivoGen), or ODN2006 (10 $\mu \mathrm{g} / \mathrm{mL}$, AdipoGen, San Diego, CA, USA), respectively. Data are presented as the mean \pm standard error of the mean. Statistical comparisons of quantitative data were carried out by the TukeyKramer test. Differences were considered to be significant when the value of $P$ was less than $0.05(P<0.05)$

excessive immune reactions mediated by endogenous TLR ligands may be involved in the development of type 1 AIP. The difference in the function of basophils activated via TLR2 and TLR4 signaling is still unclear. Basophils activated via TLR2 and/or TLR4 signaling may participate in Th2 immune response and elimination of endogenous debris $[51,52,53]$. In the future, it will be necessary to clarify the differences in the properties of basophils activated via different TLRs in order to better understand pancreatitis pathophysiology.

In conclusion, our results demonstrate that together with M2 macrophages, basophils activated via TLR signaling may also play an important role in pathophysiology of type 1 AIP.

Acknowledgements This study was partially supported by a Grantin-Aid for Scientific Research of the Ministry of Culture and Science of Japan (17K09468, 15K09052), by the Research Program on Intractable Diseases from the Ministry of Labor and Welfare of Japan, and by Grants-in-Aid from the Ministry of Education, Culture, Sports, Science and Technology of Japan from the CREST Japan Science and Technology Agency. We thank professor A-Hon Kwon for pancreatic surgery. We warmly thank Mr. Gonda for technical advice about flow cytometry data analysis.

\section{Compliance with ethical standards}

Conflict of interest The authors declare that they have no conflicts of interest.

Open Access This article is distributed under the terms of the Creative Commons Attribution 4.0 International License (http://crea tivecommons.org/licenses/by/4.0/), which permits unrestricted use, 
distribution, and reproduction in any medium, provided you give appropriate credit to the original author(s) and the source, provide a link to the Creative Commons license, and indicate if changes were made.

\section{References}

1. Okazaki K, Uchida K, Miyoshi H, et al. Recent concepts of autoimmune pancreatitis and IgG4-related disease. Clin Rev Allergy Immunol. 2011;41(2):126-38.

2. Uchida K, Okazaki K, Konishi Y, et al. Clinical analysis of autoimmune-related pancreatitis. Am J Gastroenterol. 2000;95(10):2788-94.

3. Uchida K, Miyoshi H, Ikeura T, et al. Clinical and pathophysiological issues associated with type 1 autoimmune pancreatitis. Clin J Gastroenterol. 2016;9(1):7-12.

4. Sarles H, Sarles JC, Muratore R, et al. Chronic inflammatory sclerosis of the pancreas-an autonomous pancreatic disease? Am J Dig Dis. 1961;6:688-98.

5. Kawaguchi K, Koike M, Tsuruta K, et al. Lymphoplasmacytic sclerosing pancreatitis with cholangitis: a variant of primary sclerosing cholangitis extensively involving pancreas. Hum Pathol. 1991;22(4):387-95.

6. Yoshida K, Toki F, Takeuchi T, et al. Chronic pancreatitis caused by an autoimmune abnormality. Proposal of the concept of autoimmune pancreatitis. Dig Dis Sci. 1995;40(7):1561-8.

7. Hamano H, Kawa S, Horiuchi A, et al. High serum IgG4 concentrations in patients with sclerosing pancreatitis. N Engl J Med. 2001;344(10):732-8.

8. Notohara K, Burgart LJ, Yadav D, et al. Idiopathic chronic pancreatitis with periductal lymphoplasmacytic infiltration: clinicopathologic features of 35 cases. Am J Surg Pathol. 2003;27(8):1119-27.

9. Shimosegawa T, Chari ST, Frulloni L, et al. International consensus diagnostic criteria for autoimmune pancreatitis: guidelines of the International Association of Pancreatology. Pancreas. 2011;40(3):352-8.

10. Deshpande V, Zen Y, Chan JK, et al. Consensus statement on the pathology of IgG4-related disease. Mod Pathol. 2012;25(9):1181-92.

11. Umehara H, Okazaki K, Masaki Y, et al. A novel clinical entity, IgG4-related disease (IgG4RD): general concept and details. Mod Rheumatol. 2012;22(1):1-14.

12. Okazaki K, Kawa S, Kamisawa $\mathrm{T}$, et al. Japanese clinical guidelines for autoimmune pancreatitis. Pancreas. 2009;38(8):849-66.

13. Kamisawa T, Egawa N, Nakajima H. Autoimmune pancreatitis is a systemic autoimmune disease. Am J Gastroenterol. 2003;98(12):2811-2.

14. Masaki Y, Dong L, Kurose N, et al. Proposal for a new clinical entity, IgG4-positive multiorgan lymphoproliferative syndrome: analysis of 64 cases of IgG4-related disorders. Ann Rheum Dis. 2009;68(8):1310-5.

15. Umemura T, Zen Y, Hamano H, et al. Immunoglobin G4-hepatopathy: association of immunoglobin G4-bearing plasma cells in liver with autoimmune pancreatitis. Hepatology. 2007;46(2):463-71.

16. Miyoshi H, Uchida K, Taniguchi $\mathrm{T}$, et al. Circulating naive and $\mathrm{CD} 4+\mathrm{CD} 25$ high regulatory $\mathrm{T}$ cells in patients with autoimmune pancreatitis. Pancreas. 2008;36(2):133-40.

17. Tanaka A, Moriyama M, Nakashima $\mathrm{H}$, et al. Th2 and regulatory immune reactions contribute to IgG4 production and the initiation of Mikulicz disease. Arthr Rheumtol. 2012;64(1):254-63.
18. Kusuda T, Uchida K, Miyoshi H, et al. Involvement of inducible costimulator-and interleukin 10-positive regulatory $\mathrm{T}$ cells in the development of IgG4-related autoimmune pancreatitis. Pancreas. 2011;40(7):1120-30.

19. Uchida K, Tanaka T, Gershwin ME, et al. The geoepidemiology and clinical aspects of IgG4-related disease. Semin Liver Dis. 2016;36(3):187-99.

20. Fukui Y, Uchida K, Sakaguchi Y, et al. Possible involvement of Toll-like receptor 7 in the development of type 1 autoimmune pancreatitis. J Gastroenterol. 2015;50(4):435-44.

21. Moriyama M, Tanaka A, Maehara T, et al. T helper subsets in Sjogren's syndrome and IgG4-related dacryoadenitis and sialoadenitis: a critical review. J Autoimmun. 2014;51:81-8.

22. Detlefsen S, Sipos B, Zhao J, et al. Autoimmune pancreatitis: expression and cellular source of profibrotic cytokines and their receptors. Am J Surg Pathol. 2008;32(7):986-95.

23. Egawa M, Mukai K, Yoshikawa S, et al. Inflammatory monocytes recruited to allergic skin acquire an anti-inflammatory M2 phenotype via basophil-derived interleukin-4. Immunity. 2013;38(3):570-80.

24. Falcone FH, Haas H, Gibbs BF. The human basophil: a new appreciation of its role in immune responses. Blood. 2000;96(13):4028-38.

25. Devouassoux G, Foster B, Scott ML, et al. Frequency and characterization of antigen-specific IL4 and IL13 producing basophils and $\mathrm{T}$ cells in peripheral blood of healthy and asthmatic subjects. J Allergy Clin Immunol. 1999;104:811-9.

26. Schroeder JT, MacGlashan DW Jr, Lichtenstein LM. Human basophils: mediator release and cytokine production. Adv Immunol. 2001;77:93-122.

27. Karasuyama H, Mukai K, Tsujimura Y, et al. Newly discovered roles for basophils: a neglected minority gains new respect. Nat Rev Immunol. 2009;9(1):9-13.

28. Obata K, Mukai K, Tsujimura Y, et al. Basophils are essential initiators of a novel type of chronic allergic inflammation. Blood. 2007;110(3):913-20.

29. Ito Y, Satoh T, Takayama K, et al. Basophil recruitment and activation in inflammatory skin diseases. Allergy. 2011;66(8):1107-13.

30. Nirula A, Glaser SM, Kalled SL, et al. What is IgG4? A review of the biology of a unique immunoglobulin subtype. Curr Opin Rheumatol. 2011;23(1):119-24.

31. Italiani P, Boraschi D. From monocytes to M1/M2 macrophages: phenotypical vs. functional differentiation. Front Immunol. 2014;5:514.

32. Gordon S. Alternative activation of macrophages. Nat Rev Immunol. 2003;3(1):23-35.

33. Notohara K, Wani Y, Fujisawa M. Proliferation of CD163+ spindle-shaped macrophages in IGG4-related sclerosing disease: analysis of lymphoplasmacytic sclerosing pancreatitis and sclerosing sialadenitis. Mod Pathol. 2010;23:367A.

34. Furukawa S, Moriyama M, Tanaka A, et al. Preferential M2 macrophages contribute to fibrosis in IgG4-related dacryoadenitis and sialoadenitis, so-called Mikulicz's disease. Clin Immunol. 2015;156(1):9-18.

35. Okazaki K, Uchida K, Fukui T. Recent advances in autoimmune pancreatitis: concept, diagnosis, and pathogenesis. J Gastroenterol. 2008;43(6):409-18.

36. Louten J, Rankin AL, Li Y, et al. Endogenous IL-33 enhances Th2 cytokine production and T-cell responses during allergic airway inflammation. Int Immunol. 2011;23(5):307-15.

37. Fukuhara S, Moriyama M, Miyake K, et al. Interleukin-33 produced by M2 macrophages and other immune cells contributes to Th2 immune reaction of IgG4-related disease. Sci Rep. 2017;7:42413. 
38. Yoshimoto T. Basophils as Th2-inducing antigen-presenting cells. Int Immunol. 2010;22(7):543-50.

39. Uronen-Hansson H, Allen J, Osman M, et al. Toll-like receptor 2 (TLR2) and TLR4 are present inside human dendritic cells, associated with microtubules and the Golgi apparatus but are not detectable on the cell surface: integrity of microtubules is required for interleukin-12 production in response to internalized bacteria. Immunology. 2004;111(2):173-8.

40. Rawlings DJ, Marc A, et al. Integration of B cell responses through Toll-like receptors and antigen receptors. Nat Rev Immunol. 2012;12:282-94.

41. Xu D, Komai-Koma M, Liew FY. Expression and function of Toll-like receptor on T cells. Cell Immunol. 2005;233:85-9.

42. Watanabe T, Yamashita K, Sakurai T, et al. Toll-like receptor activation in basophils contributes to the development of IgG4related disease. J Gastroenterol. 2013;48(2):247-53.

43. Paik WH, Ryu JK, Park JM, et al. Clinical and pathological differences between serum immunoglobulin G4-positive and negative type 1 autoimmune pancreatitis. World J Gastroenterol. 2013;19(25):4031-8.

44. Mills KH. TLR-dependent T cell activation in autoimmunity. Nat Rev Immunol. 2011;11(12):807-22.

45. Clarke TB, Davis KM, Lysenko ES, et al. Recognition of peptidoglycan from the microbiota by Nod1 enhances systemic innate immunity. Nat Med. 2010;16(2):228-31.

46. Park JS, Svetkauskaite D, He Q, et al. Involvement of toll-like receptors 2 and 4 in cellular activation by high mobility group box 1 protein. J Biol Chem. 2004;279(9):7370-7.
47. Hoque R, Malik AF, Gorelick F, et al. Sterile inflammatory response in acute pancreatitis. Pancreas. 2012;41(3):353-7.

48. Schaefer L, Babelova A, Kiss E, et al. The matrix component biglycan is proinflammatory and signals through Toll-like receptors 4 and 2 in macrophages. $J$ Clin Invest. 2005;115(8):2223-33.

49. Tsung A, Sahai R, Tanaka H, et al. The nuclear factor HMGB1 mediates hepatic injury after murine liver ischemia-reperfusion. J Exp Med. 2005;201(7):1135-43.

50. Lande R, Gregorio J, Facchinetti V, et al. Plasmacytoid dendritic cells sense self-DNA coupled with antimicrobial peptide. Nature. 2007;449(7162):564-9.

51. Urbonaviciute V, Furnrohr BG, Meister S, et al. Induction of inflammatory and immune responses by HMGB1-nucleosome complexes: implications for the pathogenesis of SLE. J Exp Med. 2008;205(13):3007-18.

52. Bieneman AP, Chichester KL, Chen YH, et al. Toll-like receptor 2 ligands activate human basophils for both IgE-dependent and IgE-independent secretion. J Allergy Clin Immunol. 2005;115(2):295-301.

53. Suurmond J, Stoop JN, Rivellese F, et al. Activation of human basophils by combined toll-like receptor- and FceRI-triggering can promote Th2 skewing of naive T helper cells. Eur J Immunol. 2014;44(2):386-96. 